\title{
A Perda do Peso Morto e a Elasticidade-preço da Demanda do Setor Siderúrgico no Brasil
}

\begin{abstract}
RESUMO
Dois objetivos orientam este artigo. O primeiro foi calcular o valor da perda do bem-estar do setor siderúrgico no Brasil. Para alcançar este propósito foi preciso estimar a equação de demanda por aço para, então, encontrar a sua elasticidade-preço. Conseqüentemente, este passou a ser o segundo objetivo do presente ensaio. $O$ estudo aqui apresentado é o primeiro, do ponto de vista quantitativo, para o Brasil, no que concerne aos dois fins, e seus resultados sugerem que: primeiro, a elasticidade é de -0, I 4 e, segundo, a perda de bem-estar social no setor siderúrgico em 2000 e 200 I, devido ao poder de monopólio das firmas, é de aproximadamente $24 \%$, em média, com relação ao faturamento da indústria ou $0,30 \%$ com relação ao produto interno bruto (PIB).
\end{abstract}

\section{Palavras-CHAVE}

perda do bem-estar, função de demanda, elasticidade-preço da demanda, siderurgia

\section{ABSTRACT}

This article has two objectives. The first one is to calculate the deadweight loss of Brazil's steel sector. It was necessary, for this purpose, to estimate the steel demand's equation for, therefore, derivate its price-elasticity. This is, consequently, the article's second objective. The study proposed here is the first one, concerning the two objectives and regarding to quantitative measures, to Brazil. The results suggests that: first, the price-elasticity is $-0,14$ and, second, the deadweight loss for the steel sector (2000 and $200 \mathrm{I}$ ) is, in average, approximately, of $24 \%$ of total sector's revenue or $0,30 \%$ of the GDP.

\section{KEY WORDS}

deadweight loss, demand's function, price-elasticity of demand, siderurgy

\section{JEL Classification}

L6IO, LI20

\footnotetext{
* Doutora em economia pela EPGE/FGV/RJ, ex-secretária adjunta do Ministério da Fazenda (SEAE) e economista chefe da Compañia de Comercio y Exportación de Puerto Rico. Email: schmidt@prtc.net. Endereço para contato: Rua Ipanema 53/I60I, Condomínio Nova Ipanema. Barra da Tijuca - Rio de Janeiro - RJ, Brasil. CEP 2263 I-390.

* * Professor do IBMEC-RJ e técnico da Secretaria de Acompanhamento Econômico do Ministério da Fazenda. E-mail: mamdl@uol.com.br. Endereço para contato: Av. Presidente Antonio Carlos 375, sala 1038. Centro - Rio de Janeiro - RJ. CEP 20020-0I0.

(Recebido em abril de 2004. Aceito para publicação em março de 2005)
} 


\section{INTRODUÇÃO}

A indústria siderúrgica brasileira estrutura-se segundo um oligopólio, assim como ocorre com a maior parte das indústrias do País e conforme se observa em nível internacional. É, portanto, um mercado que comporta poucas firmas. Além disso, existem elevadas barreiras à entrada, há pouca importação e não são muitos os produtos substitutos do aço. Estes fatores indicam que a elasticidade-preço da demanda deve ser baixa e que a rivalidade entre as firmas deve ser pequena, até porque nem todas as siderúrgicas produzem todos os tipos de aço. Tais características corroboram a hipótese de que essas firmas não só tenham elevado poder de mercado, mas também a possibilidade de exercê-lo. Se isso ocorre, quem perde é o consumidor, e as firmas são as ganhadoras.

Estimar a elasticidade-preço da demanda e calcular o valor da perda do bem-estar $(\mathrm{DWL})^{1}$ desse mercado são, assim, os objetivos do presente artigo. Não se tem conhecimento de qualquer estudo quantitativo deste tipo para o Brasil. Além disso, um dos poucos casos de condenação pelo Conselho Administrativo de Defesa da Concorrência (CADE) refere-se ao mercado de aço, julgado em 1999. ${ }^{2}$

O cálculo do DWL é relevante porquanto mensura a perda monetária que uma determinada comunidade sofre devido ao exercício do poder de mercado de uma empresa ou de uma indústria. A importância, entretanto, não está em fornecer informações a todos acerca da magnitude desta perda, mas, sobretudo, fazer com que os resultados encontrados sirvam de insumo para a formulação de políticas públicas que visem fomentar uma maior concorrência no mercado, principalmente por parte dos órgãos antitruste do País, que constituem o Sistema Brasileiro de Defesa da Concorrência (SBDC). ${ }^{3}$ Estes órgãos, grosso modo, analisam os impactos no bem-estar social, tanto no que concerne a "fusões e aquisições" quanto no referente às condutas anticompetitivas realizadas pelas empresas. Além disso, como o caso condenado pelo CADE, em 1999, relativo ao mercado de aço continua ainda sob análise no Sistema Judiciário, este estudo poderia servir como fonte documental para se chegar a uma conclusão mais rápida sobre o mesmo.

Por outro lado, a estimação da função de demanda do aço foi necessária para não só servir de insumo para o cálculo do DWL, por não haver qualquer trabalho com tal es-

1 DWL é a abreviação usual para Deadweight Loss.

2 O processo de cartel julgado pelo CADE, envolvendo as empresas CSN, Usiminas e a COSIPA, é o de número 08000.015337/97-48. Elas foram julgadas em 1999 e o caso ainda está sendo analisado pelo Sistema Judiciário.

3 O Sistema Brasileiro de Defesa da Concorrência (SBDC) é composto pela Secretaria de Acompanhamento Econômico (SEAE), pela Secretaria de Direito Econômico (SDE) e pelo Conselho Administrativo de Defesa da Concorrência (CADE). 
timação para o Brasil, mas, sobretudo, para proporcionar um parâmetro para futuros estudos ou para possíveis políticas públicas com relação ao mercado siderúrgico.

O artigo de Harberger (1954) foi o pioneiro na estimação do valor da perda do bemestar. Seu estudo enfocou o setor manufatureiro nos EUA. Mais tarde, seguindo a sua metodologia, pesquisadores na área de organização industrial fizeram a estimação para outros mercados. A teoria recente, baseada em um modelo de oligopólio, e não em uma extensão de monopólio como discutida em Harberger (1954), é apresentada em Daskin (1991). O presente trabalho também se baseia nestes princípios teóricos. No atinente à estimação da função de demanda, o artigo segue os modelos tradicionais sobre o tema (independente do setor). A especificação da função, em particular, foi motivada pelo trabalho de Abbot et al. (1999).

O texto está estruturado da seguinte forma: na seção 1 apresenta-se um resumo da literatura; na seção 2, o modelo teórico para o cálculo do DWL e para a estimação da equação de demanda de aço; na seção 3 são expostos os dados utilizados e a adaptação empírica ao modelo teórico para o setor siderúrgico brasileiro; na seção 4, expõem-se os resultados tanto do cálculo do DWL quanto da estimação da demanda; por último, apresentam-se as conclusões.

\section{REVISÃO DA LITERATURA ${ }^{4}$}

Duas importantes correntes na temática organização industrial tiveram início com os trabalhos de Bain (1951) e de Harberger (1954). O primeiro autor iniciou os estudos na linha "estrutura-conduta-desempenho", que tem como objetivo principal encontrar a relação entre o desempenho das firmas (lucro) e a estrutura do mercado à qual pertencem, em que esta relação passaria pela conduta (colusiva) das mesmas. O segundo, no qual este ensaio se insere, almeja, genericamente, mensurar a perda, para a sociedade, de um mercado cuja estrutura não fosse competitiva.

Pode-se dizer que desde 1976 ambas as correntes passaram a calcar seus modelos a partir do modelo de Cowling e Waterson (1976). Os autores apresentaram uma expressão para o índice de Lerner, que posteriormente foi aperfeiçoada no que concerne à variação conjectural. Dickson (1981) e Clarke e Davis (1982), por exemplo, propuseram uma nova parametrização para o termo da variação conjectural de Cowling e Waterson (1976). Dixit e Stern (1982), Geroski et al. (1985), Bershanhan (1989) e Daskin (1991) procuraram calcular a perda do bem-estar social tanto teórica quanto empiricamente. ${ }^{5}$

4 Para uma descrição mais precisa, ver Sherer e Ross (1990).

5 Uma boa resenha pode ser encontrada em Frase (1994), segundo Wyllie (2001, p. 27). 
A medida de DWL, em particular, foi inicialmente elaborada por Harberger (1954), que pressupõe uma estrutura de mercado de monopólio em que a firma monopolista possui custo marginal constante e defronta-se com uma curva de demanda com elasticidade unitária. O DWL é representado pela diferença entre a redução no excedente do consumidor e o aumento no excedente do produtor, quando o mercado deixa de ser competitivo e passa a ser monopolista. Esta área pode ser calculada a partir da seguinte fórmula,

$$
D W L=\frac{1}{2} \Delta p \cdot \Delta q
$$

apontada por Harberger (1954), onde $\Delta \mathrm{p}=\mathrm{p}_{\mathrm{m}}-\mathrm{p}_{\mathrm{cp}}$ e $\Delta_{\mathrm{q}}=\mathrm{q}_{\mathrm{m}}-\mathrm{q}_{\mathrm{cp}}$, que pode ser encontrada, com detalhes, inclusive ilustração gráfica, em diversos livros-textos de microeconomia. ${ }^{6}$ Anos mais tarde, no entanto, Daskin (1991) apresentou uma fórmula de cálculo mais elaborada, que diz respeito à perda de peso morto de um mercado em oligopólio, qual seja:

$$
D W L=\frac{1}{2} \pi^{2} \eta \cdot R^{-1}
$$

Fergunson e Fergunson (1994) apresentaram, três anos depois, uma outra versão para a expressão (2), que é:

$$
D W L=\frac{1}{2}\left(\frac{\Delta p}{p}\right)^{2} \eta \cdot R
$$

Em ambas as fórmulas, (2) e (3), $\eta$ representa o valor absoluto da elasticidade-preço da demanda (não necessariamente igual a um), $\pi$ é o lucro do monopolista e $\mathrm{R}$ a receita total (ou faturamento) da firma monopolista. ${ }^{7}$

A partir da fórmula (1), Harberger (1954) calculou o DWL para o setor manufatureiro dos Estados Unidos, como porcentual do PIB, e concluiu que seu valor era de cerca de $0,08 \%$, relativamente baixo. As principais críticas a este modelo estão relacionadas às simplificações propostas por Harberger (1954) para adaptação ao modelo empírico. A primeira delas refere-se à fixação do valor da elasticidade-preço da demanda em 1 para todas as indústrias. As outras dizem respeito ao cálculo utilizado para a determinação do lucro do monopolista, e à falha do autor ao deixar de considerar a interdependência entre $\Delta \mathrm{p}$ e $\Delta \mathrm{q}$.

6 Como, por exemplo, em Varian (1992) e Mas-Colell et al. (1995).

7 As equivalências entre as equações (2) e (3) podem ser encontradas no apêndice deste artigo. 
Cowling e Muller $(1978$; 1981) tentam resolver estes problemas relaxando as hipóteses restritivas do modelo de Harberger (1954). Além disso, estimam o DWL para firmas, ao invés de realizarem este cálculo para o setor como um todo. Utilizando esta metodologia, os autores encontram valores consideravelmente maiores para os DWL, como porcentual do PIB, variando de $0,4 \%$ a $3,96 \%$, dependendo da elasticidade-preço da demanda considerada.

Mesmo tendo corrigido alguns dos problemas do modelo original, Cowling e Muller (1978; 1981) continuavam a basear seus cálculos do DWL em um modelo cuja estrutura de mercado era a de monopólio. Holt (1982) alega que os modelos mencionados só são apropriados para estruturas de mercado monopolísticas com demanda linear e custos marginais constantes. Eles não seriam apropriados para calcular o DWL proveniente de uma estrutura de mercado oligopolizada. ${ }^{8}$ Além disso, Holt (1982) e Masson e Shaanan (1984) concluem que é mais apropriado utilizar um modelo baseado na indústria do que em firmas individuais.

Dixit e Stern (1982), ao estimarem o DWL de todos os setores da economia dos EUA, obtiveram valores de até $20 \%$ do PIB. Daskin (1991), usando a parametrização de Clarke e Davis (1982) para a variação conjectural, também obtém valores elevados para o DWL da indústria do Reino Unido. Cysne et al. (2001) e Wyllie (2001) realizaram um estudo para o setor de cervejas do Brasil, ambos seguindo a linha de Daskin (1991). A conclusão sobre o DWL, relativo à receita total da indústria, foi de que este estaria entre os percentuais $20 \%$ e $50 \%$, em Cysne et al. (2001), e entre $1 \%$ e $20 \%$, em Wyllie (2001, p. 41). A diferença dos resultados entre os dois trabalhos não é clara, mas em ambos estes dependem do valor escolhido para a conduta e também da firma entendida como a mais eficiente (Antarctica, Brahma ou Kaiser).

Outros autores fizeram o cálculo do DWL para diversos setores da economia e em diferentes momentos da história. Estes são apresentados na Tabela l, a seguir, e foram apontados em Fergunson e Fergunson (1994). Um futuro estudo, portanto, poderia ser o de calcular o DWL para todos os setores da economia brasileira.

8 Mesmo idealizados para monopólios, os modelos de Harberguer (1954) e Cowling e Muller (1978; 1981) eram utilizados para calcular DWL em mercados oligopolizados. 
TABELA 1 - ESTUDOS ANTERIORES SOBRE PERDA DE PESO MORTO

\begin{tabular}{llll}
\hline Autores & Período & País & DWL/PIB (\%) \\
\hline Harberguer (1954) & $1924-28$ & EUA & 0,1 \\
Scwartzman (1960) & 1954 & EUA & 0,1 \\
Kamerschen (1966) & $1956-61$ & EUA & $5,4-7,6$ \\
Bell (1968) & 1954 & EUA & $0,02-0,04$ \\
Shepherd (1970) & $1960-69$ & EUA & $2,0-3,0$ \\
Worcester (1973) & $1956-69$ & EUA & $0,2-0,7$ \\
Siegfried e Tiemann (1974) & 1963 & EUA & 0,07 \\
Cowling and Mueller (1978) & $1963-66$ & EUA & $4,0-13,1$ \\
Masson e Shaanan (1984) & $1950-66$ & EUA & 2,9 \\
Wahlroos (1984) & $1962-75$ & EUA & $0,04-0,90$ \\
Gisser (1986) & 1977 & EUA & $0,1-1,8$ \\
Jones e Laudadio (1978) & $1965-67$ & Canadá & 3,7 \\
Jenny e Weber (1983) & $1967-70$ & França & $0,13-8,85$ \\
& $1971-74$ & França & 0,21 \\
Pezzoli (1985) & $1982-83$ & Itália & $0,4-9,4$ \\
Funahashi (1982) & 1980 & Japão & $0,02-3,00$ \\
Oh (1986) & 1983 & Coréia & $1,16-6,75$ \\
Ong'olo (1987) & 1977 & Quênia & $0,26-4,40$ \\
\hline
\end{tabular}

Fonte: Fergunson e Fergunson (1994).

\section{MODELO TEÓRICO}

\subsection{Cálculo do DWL}

O modelo aqui utilizado baseia-se em Daskin (1991), que partiu do trabalho de Cowling e Waterson (1976), e pode ser sintetizado da seguinte forma: seja a demanda com elasticidade constante (isoelástica) dada por: $Q=A P^{-\varepsilon}$, em que $\mathrm{Q}$ é a quantidade total vendida na indústria, $\mathrm{A}>0$ é uma constante, $\varepsilon$ é o valor absoluto da elasticidade preço da demanda e $\mathrm{P}$ o preço do produto homogêneo em questão. A indústria tem $\mathrm{N}$ firmas, em que $\mathrm{q}_{\mathrm{i}}$ representa a quantidade vendida pela firma i, $S_{i}=\frac{q_{i}}{Q}$ a participação de mercado (market share) da firma i e $\mathrm{C}_{\mathrm{i}}$ o custo marginal da firma i. Cada firma i resolve o seu problema individual de maximização de lucros da seguinte forma: $\Pi_{i}=R T_{i}(Q)-C T_{i}\left(q_{i}\right)$, onde $\Pi i$ é o lucro da firma i, $\mathrm{RT}_{\mathrm{i}}(\mathrm{Q})$ é a receita total (ou faturamento) da firma i e $\mathrm{CT}_{\mathrm{i}}\left(\mathrm{q}_{\mathrm{i}}\right)$ é o custo total da firma i. Por hipótese, não há custo fixo, o custo marginal $C_{i}$ é constante, podendo variar entre cada uma das firmas, e a 
variação conjectural segue a seguinte forma: $\frac{d q_{k}}{d q_{i}}=\alpha_{i} * \frac{q_{k}}{q_{i}}, \forall k \neq \mathrm{i}, \alpha_{\mathrm{i}} \in(0,1] . \mathrm{Da}$ condição de primeira ordem chega-se ao seguinte resultado: ${ }^{9}$

$$
P(Q)\left\{1-\frac{\left[S_{i}\left(1-\alpha_{i}\right)+\alpha_{i}\right]}{\varepsilon}\right\}-C_{i}=0
$$
Seja o índice de Lerner: ${ }^{10} L_{i}=\frac{P(Q)-C_{i}}{P(Q)}$. A equação acima pode, assim, ser reescrita
da seguinte forma:

$$
L_{i}=\frac{\left[S_{i}\left(1-\alpha_{i}\right)+\alpha_{i}\right]}{\varepsilon}
$$

De acordo com a equação (5), se a firma tiver uma participação de mercado elevada, ou se a elasticidade da demanda em valor absoluto for baixa, ou se a variação conjectural for alta, o poder de mercado da firma i aumentará.

De acordo com Mas Collel et al. (1995), a definição formal para o DWL consiste na redução do bem-estar provocada pela distorção da quantidade com respeito ao equilíbrio competitivo. Em termos analíticos a variação pode tomar a seguinte forma:

$$
D W L=\int_{P_{C P}}^{P_{O}}\{Q(P)-C m g[Q(P)]\} d P
$$

A primeira parte da integral refere-se ao excedente do consumidor (EC), e a segunda, ao excedente do produtor (EP). Admitindo, como já mencionado, uma demanda isoelástica, Daskin (1991) chega às seguintes expressões para o EC e para o EP: ${ }^{11}$

$$
\begin{aligned}
& E P=R T(Q)_{o} * \sum_{i=1}^{N} L_{i} * S_{i} \\
& E C=\frac{R T(Q)_{O}}{(1-\varepsilon)}\left[1-\left(1-L_{i}\right)^{(1-\varepsilon)}\right]
\end{aligned}
$$

9 O cálculo da condição de primeira ordem pode ser visto no apêndice deste artigo.

10 Ver em Mas-Collel et al. (1995) ou em Varian (1992). Este índice também é chamado de Price-Cost Margin. A fórmula (5) pode ser também encontrada, com mais detalhes, em Wyllie (2001).

11 Os cálculos estão apresentados no apêndice deste ensaio. 
Pelos cálculos de Daskin (1991), quando o equilíbrio é o de concorrência perfeita $\left(\mathrm{Q}_{\mathrm{cp}}, \mathrm{P}_{\mathrm{cp}}\right)$, como $\mathrm{L}_{\mathrm{i}}=0 \forall \mathrm{i}=1, \ldots, \mathrm{N}, \mathrm{EP}=0$, e quando o equilíbrio é o de oligopólio $\left(\mathrm{Q}_{0}, \mathrm{P}_{\mathrm{o}}\right), \mathrm{EC}=0$. A variação do excedente total $(\Delta \mathrm{ET})$, que é o $\mathrm{DWL}$ desta forma, é DWL = EC - EP. A curva de oferta, neste caso (diferentemente de Harberger (1954), que baseia seu cálculo em um modelo de monopólio), é crescente em formato de escada, porque, por hipótese, a firma com o menor custo, $\mathrm{C}^{*}=\mathrm{P}_{\mathrm{cp}}$, vende as primeiras unidades do produto; a firma com o segundo menor custo vende a segunda parte, e assim sucessivamente, até que a curva de oferta atinja a curva de demanda de mercado.

\subsection{Estimação da Elasticidade-preço da Demanda}

Há, pelo menos, três metodologias para estimar uma função de demanda: por equações simultâneas, por análise de co-integração, se possível, e se não for possível, por variáveis instrumentais. Com relação à especificação da função de demanda, esta foi motivada pelo estudo de Abbot et al. (1999), ${ }^{12}$ que estimou esta função para o Reino Unido. Os autores concluíram que para o setor siderúrgico naquele país a elasticidadepreço da demanda é de $-0,62$.

Foram realizados em todas as séries testes de raiz unitária ${ }^{13}$ com o intuito de verificar se eram estacionárias. Se as séries fossem não-estacionárias e possuíssem a mesma ordem de integração, seria apropriado realizar testes de co-integração para verificar a existência, entre elas, de uma relação de longo prazo que fosse estacionária. ${ }^{14}$ Técnicas que não levem em consideração a não-estacionariedade das séries poderiam levar a resultados viesados. Caso contrário, ou seja, se as séries fossem estacionárias, a função deveria ser estimada pelo método dos mínimos quadrados ordinários - MQO (caso as variáveis do lado direito fossem todas exógenas) ou pelo método das variáveis instrumentais - VI (caso houvesse alguma variável endógena do lado direito da equação). No presente caso, como se trata de uma função de demanda, o segundo método é mais apropriado, uma vez que o preço é uma variável endógena.

Foi adotada uma função do tipo Cobb-Douglas para representar a demanda dos indivíduos. Aplicando-se logaritmo natural $(\mathrm{Ln})$ à função, obteve-se o modelo a ser estima-

12 Foram pesquisados outros estudos sobre este assunto no ECOLIT e em alguns outros sites na internet, mas nada foi encontrado. Mendes de Paula (2002), no entanto, aponta em seu trabalho que a elasticidade-preço da demanda por aço obtidas para outros países encontra-se entre $-0,25$ e $-0,70$, sendo este último valor pouco provável.

13 O teste realizado foi o de Dickey Fuller Aumentado (ADF). Ver em Enders (1995, capítulo 4).

14 Neste caso, seriam realizados dois testes de co-integração: um, de acordo com Engle e Granger (1987), e o segundo, de acordo com o procedimento que segue o modelo de Johansen (1988 e 1991) e Johansen e Juselius (1990). 
do, apresentado nas equações (8) e (9), a seguir. Ao usar esta especificação considerouse que as elasticidades são constantes ao longo da curva de demanda.

$$
\begin{aligned}
& \operatorname{Ln}(Q)=\alpha_{0}+\alpha_{1} \operatorname{Ln}(P)+\alpha_{3} \operatorname{Ln}(P I B)+\alpha_{4} \operatorname{Ln}(P A)+\alpha_{4} \operatorname{Ln}(C C)+\alpha_{5} \operatorname{Ln}(P I) \\
& q=\alpha_{0}+\alpha_{1} p+\alpha_{3} p i b+\alpha_{4} p a+\alpha_{4} c c+\alpha_{5} p i
\end{aligned}
$$

onde Q representa a quantidade vendida de aço bruto; P o preço do aço; PIB é o produto interno bruto; PA a produção de automóveis; CC, um índice que mede a atividade da construção civil, e PI, um índice que mede a produção industrial. As variáveis da equação (9) dizem respeito, respectivamente, às variáveis anteriores quando aplicado o Ln.

\section{DADOS UTILIZADOS E ADAPTAÇÃO EMPÍRICA}

\subsection{Dados Utilizados}

Para o cálculo do DWL foi usada uma amostra englobando dez empresas do setor siderúrgico brasileiro, como pode ser visto no apêndice deste ensaio. Todas atuam em produtos que derivam de aços planos ou de aços longos. Calculou-se o DWL para os anos de 2000 e 2001, com e sem importação e para dois tipos de aço, quanto à forma geométrica: ${ }^{15}$ aços planos, aços longos e vergalhão. Este último tipo de aço corresponde, aproximadamente, a 30\% do faturamento da categoria aço longo e é de muita importância para a construção civil.

As importações foram obtidas no Anuário Estatístico do Instituto Brasileiro de Siderurgia (IBS) de 2002. Os preços praticados e as quantidades vendidas por tipo de aço foram fornecidos pelas próprias empresas do setor siderúrgico. A partir destes dados foram calculados os faturamentos (RT) em cada um dos mercados. As participaçóes de mercado das empresas $(\mathrm{Si})$, nos três distintos mercados, foram obtidas pela razão entre a quantidade vendida pela empresa i (em toneladas) e a quantidade vendida total, e, também, pela razão do faturamento da firma i e o faturamento total. O índice de Lucro (Li) foi obtido da seguinte forma: com os dados de Receita Operacional Líquida (ROL) e Lucro Líquido (LL), calculou-se o índice LLROL (=LL/ROL). Estas variáveis foram encontradas na base de dados que a Comissão de Valores Mobiliários (CVM) mantém em seu site na internet. O PIB foi obtido no IPEA, em seu site na internet. ${ }^{16}$ Por fim, foi estimada a elasticidade-preço da demanda $(\varepsilon)$.

15 No site do Instituto Brasileiro de Siderurgia (IBS) é possível encontrar um bom resumo sobre cada tipo. 16 www.ipeadata.gov.br. 
Para a estimação da equação de demanda de aço no Brasil foram utilizados dados mensais do período de 1981 a 2001. As variáveis utilizadas foram: a quantidade produzida de aço bruto no Brasil, encontrada no anuário do IBS; o preço do aço (IPA-OG ferro, aço e derivados), calculado pela Fundação Getúlio Vargas (FGV), o qual foi deflacionado pelo IPA-DI (divulgado pela FGV); a produção de automóveis, fornecida pela Associação Nacional dos Fabricantes de Veículos Automotores (Anfavea); a renda (PIB - preços de mercado), calculada pelo IPEA; o índice que mede o nível de atividade na construção civil (Produção Industrial - insumo - construção civil - quantum), fornecido pelo Instituto Brasileiro de Geografia e Estatística, Pesquisa Mensal Produção Física (IBGE Outras/PIM - PF nova); o índice que mede o nível de produção da indústria geral, fornecido pelo IBGE (Produção industrial da indústria geral: índice de quantum), também fornecido pelo IBGE Outras/PIM - PF; e o preço internacional do aço, fornecido pelo IBS, cuja fonte primária é o "Metal Bulletin", o qual foi deflacionado pelo CPI-W (Consumer Price Index produzido pelo Burean of Labor Statistics - BLS).

Houve duas adaptações neste trabalho. A primeira, seguindo métodos recorrentes neste tipo de estimação, sugerida em Daskin (1991), foi a utilização, como critério de escolha da firma de menor custo, $C^{*}=\operatorname{Min}_{i}^{*}=P(Q)_{C P}$, aquela que tivesse o maior lucro. Isto porque, devido à assimetria de informação sobre o custo das empresas uma vez que não se tem acesso a todos os dados da empresa -, o seu cálculo pode gerar graves distorções. A segunda adaptação concerne à medida de lucro utilizada. Esta, além de refletir o lucro contábil, diz respeito à empresa como um todo, não havendo separação por linha de produto, que seria a informação ideal.

\section{RESULTADOS}

\subsection{Estimação da Demanda de Aço no Brasil}

Como se pode observar na Tabela 2 , a seguir, cada série é $\mathrm{I}(0),{ }^{17}$ isto é, não se pode rejeitar a hipótese nula de haver raiz unitária, exceto $\mathrm{Q}$ (para todos os níveis de significância estatística) e $\mathrm{P}$ (para os níveis de $5 \%$ e 10\%). Nas demais séries, ao nível de significância de $10 \%$, pode-se rejeitar tal hipótese. Não seria apropriado, desta forma, realizar testes de co-integração. Poder-se-ia optar, portanto, pela estimativa da equação de demanda por aço pelo método MQO ou pelo método das VI.

17 Há vários livros sobre este assunto, em particular, Greene (1997) ou Vasconcelos et al. (2000). 
TABELA 2 - RESULTADOS DOS TESTES DE RAIZ UNITÁRIA (ADF)

\begin{tabular}{cclcccc}
\hline & & & Valor crítico & \multicolumn{2}{c}{ Valores críticos tabela } \\
\cline { 5 - 6 } Variável & Defasagem & Termos da equação & (teste ADF) & $1 \%^{*}$ & $5 \%$ & $10 \%$ \\
\hline Q & 1 & Constante e tendência & $-2,654081$ & $-3,998000$ & $-3,429000$ & $-3,137700$ \\
P & 2 & Constante & $-2,746012$ & $-3,458200$ & $-2,873200$ & $-2,572900$ \\
PIB & 3 & Constante e tendência & $-6,441533$ & $-3,998300$ & $-3,429200$ & $-3,137800$ \\
PA & 1 & Constante e tendência & $-5,189615$ & $-3,998000$ & $-3,429000$ & $-3,137700$ \\
CC & 2 & Constante e tendência & $-5,573436$ & $-3,998100$ & $-3,429100$ & $-3,137700$ \\
PI & 3 & Constante e tendência & $-7,434467$ & $-3,998300$ & $-3,429200$ & $-3,137800$ \\
\hline
\end{tabular}

* Valores críticos de MacKinnon para teste ADF de raíz unitária.

Além do fato de haver estacionariedade na maioria das séries em nível, como a variável endógena "preço" está do lado direito da equação (9), o método das VI é o mais apropriado para estimar esta equação. $\mathrm{O}$ instrumento proposto, além das variáveis exógenas, foi o preço internacional do aço, sendo os resultados obtidos da estimação apresentados na Tabela 3, a seguir:

\section{TABELA 3 - RESULTADOS DA ESTIMAÇÃO DA EQUAÇÃO DE DEMANDA $D E A C ̧ O$}

\begin{tabular}{lcccc}
\hline Variável & Coeficiente & $\begin{array}{l}\text { Desvio } \\
\text { padrão }\end{array}$ & Estatística - t & P-valor \\
\hline P & $-0,1403$ & 0,0107 & $-13,1398$ & 0,0000 \\
PIB & 1,0905 & 0,0873 & 12,4917 & 0,0000 \\
PA & $-0,0309$ & 0,0287 & $-10,7817$ & 0,2820 \\
CC & $-0,6987$ & 0,1293 & $-54,0332$ & 0,0000 \\
PI & 0,5649 & 0,1269 & 44,5294 & 0,0000 \\
\hline$R^{2}$ & 0,784782 & Estatística F & & 280,212 \\
$R^{2}$ Ajust. & 0,781296 & P-Valor (Est. F) & & 0,0000 \\
núm. obs & 252 & Soma dos quad. Res. & 2,9387 & \\
Instrumentos = C, PIA, PIB, PA, CC, PI & & & \\
\hline
\end{tabular}

Da tabela acima pode-se observar que a elasticidade-preço da demanda por aço no Brasil tem o sinal negativo esperado e é da ordem (em módulo) de $\mathbf{0 , 1 4}$, sugerindo certa inelasticidade com relação ao preço da demanda no Brasil. Este valor está um pouco abaixo de 0,25, um patamar inferior ao que Mendes de Paula (2002) apresenta em seu artigo, mas dentro do previsto, uma vez que o setor é concentrado e apresenta pouca 
rivalidade. Por isso, como mencionado no início do artigo, com alta probabilidade de nele haver o "exercício do poder de mercado das firmas".

Como se pode notar, PA tem o sinal não esperado, mas seu coeficiente mostrou-se estatisticamente não significativo. Uma nova estimação foi feita retirando esta variável, porém os resultados pouco se alteraram. A elasticidade-preço da demanda, por exemplo, foi de $-0,135969$. O outro sinal não esperado, mas estatisticamente significativo, ocorreu com o índice relativo à construção civil. A constante também foi retirada, pois mostrou-se estatisticamente não significativa. $\mathrm{O}$ modelo foi estimado utilizando a matriz de White para correção de problemas de heteroscedasticidade. ${ }^{18} \mathrm{O}$ resultado daquela elasticidade será utilizado no cálculo do DWL. Para modelos sem constante é sabido que o $\mathrm{R}^{2}$ pode assumir valores fora do seu domínio tradicional (entre 0 e 1 ). Com isso, deve-se ter cuidado ao interpretar o valor obtido para esta estatística nesta equação. (Greene, 1997, p. 36).

\subsection{Cálculo da Perda do Bem-estar}

Para a realização do cálculo do DWL do setor siderúrgico preferiu-se segmentar o mercado de aço em dois - aços planos e aços longos -, uma vez que os participantes do mercado (players) de um tipo não são necessariamente os mesmos que os do outro. Além disso, como o mercado do vergalhão (que pertence ao grupo dos aços longos) representa quase $30 \%$ do faturamento da sua categoria (aços longos), e por ser extremamente concentrado e importante para a construção civil, decidiu-se computar, também, o DWL deste segmento.

Duas observações devem ser feitas. A primeira é que a empresa Açominas faz parte do Grupo Gerdau desde 1998. Assim sendo, elas estão sendo entendidas, do ponto de vista antitruste, como uma única empresa, conforme se pode notar na tabela relativa à lista das empresas. A segunda é que no ano 2000 a Belgo Mineira e a CSN tiveram os seus LLROL (lucro líquido/receita operacional líquida) excepcionalmente elevados, o que resultou na necessidade de se realizar um ajuste nestes valores, pois do contrário os DWLs seriam superestimados. O critério de ajuste foi comparativo com os demais LLROL de cada segmento.

No primeiro caso, este lucro majorado ocorreu porque no balanço de 2000 da Belgo Mineira foi computada a venda das mineradoras Samitre e Samarco. Já no segundo, o grande lucro da CSN derivou do descruzamento acionário entre ela e a Companhia Vale do Rio Doce, ocorrido em 1999 e contabilizado em 2000.

18 Mais detalhes em Greene (1997, capítulo 12). 
A CSN e a Gerdau foram, em 2000 e em 2001, as empresas que apresentaram o maior LLROL no mercado de aços planos. Já no dos aços longos e no dos vergalhões, em particular, foram as empresas Belgo-Mineira e Barra Mansa. Observa-se que a escolha da CSN e da Belgo Mineira foi mantida mesmo com o ajuste no LLROL de cada uma para o ano de 2000 .

A Tabela 4, a seguir, apresenta os resultados dos cálculos de DWL para cada um dos três mercados nos anos de 2000 e de 2001. A medida está relativizada tanto pelo faturamento de cada mercado (DWL/Fat.) quanto pelo produto interno bruto do País (DWL/PIB) de cada ano.

TABELA 4 - CÁLCULOS DWL/FATURAMENTO E DWL/PIB

\begin{tabular}{|c|c|c|c|c|c|}
\hline & \multicolumn{2}{|c|}{ Sem Importação } & \multicolumn{2}{|c|}{ Com Importação } & \multirow[b]{2}{*}{ Média } \\
\hline & 2000 & 2001 & 2000 & 2001 & \\
\hline \multicolumn{6}{|c|}{ Aços Planos } \\
\hline DWL/Fat. & $22,50 \%$ & $20,33 \%$ & $23,10 \%$ & $19,65 \%$ & $21,39 \%$ \\
\hline DWL/PIB & $0,167 \%$ & $0,148 \%$ & $0,184 \%$ & $0,154 \%$ & $0,16 \%$ \\
\hline \multicolumn{6}{|c|}{ Aços Longos } \\
\hline DWL/Fat. & $25,22 \%$ & $26,34 \%$ & $26,25 \%$ & $28,45 \%$ & $26,57 \%$ \\
\hline DWL/PIB & $0,134 \%$ & $0,136 \%$ & $0,147 \%$ & $0,155 \%$ & $0,14 \%$ \\
\hline \multicolumn{6}{|c|}{ Vergalhões } \\
\hline DWL/Fat. & $33,09 \%$ & $27,63 \%$ & $34,64 \%$ & $29,12 \%$ & $31,12 \%$ \\
\hline DWL/PIB & $0,047 \%$ & $0,047 \%$ & $0,051 \%$ & $0,052 \%$ & $0,05 \%$ \\
\hline \multicolumn{4}{|c|}{ DWL índustria (média 2000 e 2001 em R\$) } & & \\
\hline \multicolumn{3}{|c|}{ Faturamento indústria (média 2000 e 2001 em R\$) } & 14.958.646.120,06 & & \\
\hline \multicolumn{3}{|c|}{ PIB médio $(\mathrm{R} \$)$} & 1.149.995.633.000,00 & & \\
\hline \multicolumn{3}{|c|}{ DWL/Fat. } & $\begin{array}{r}23,52 \% \\
031 \%\end{array}$ & & \\
\hline \multicolumn{3}{|l|}{ DWL/PIB } & $0,31 \%$ & & \\
\hline
\end{tabular}

A Tabela 4 possibilita levantar alguns pontos. $\mathrm{O}$ primeiro diz respeito à magnitude do DWL, que não é desprezível em nenhum dos três segmentos, todos acima de $20 \%$, com relação ao faturamento de seus respectivos mercados.

O segundo é que, comparativamente, o mercado de vergalhões apresenta o maior DWL (31\% com referência ao faturamento deste mercado), seguido pelo mercado de aços longos, com 26\%, que, por sua vez, é seguido pelo mercado de aços planos, com $22 \%$. Se for tomada a média ponderada no setor siderúrgico, o DWL/Faturamento é de $24 \%$ e o DWL/PIB é de $0,31 \%$. Em outras palavras, devido à falta de competição no setor, a sociedade como um todo perde aproximadamente R $\$ 3,5$ bilhões (a preços médios de 2000 e 2001). Observe-se que a interpretação, do ponto de vista do PIB, não é a mais intuitiva, quando se comparam as médias entre os três segmentos, pois não se leva em consideração a representatividade de cada mercado (isto é, o tamanho 
de cada um). Esta informação, não obstante, é importante, pois ela, "ao fim e ao cabo", mensura a perda, para a sociedade, da economia inteira.

Este fato era de alguma forma esperado, quando se observam os market shares ${ }^{19} \mathrm{e}$ as concentrações de mercado, apresentados no apêndice deste estudo. $\mathrm{O}$ mercado de vergalhão, por exemplo, tem o Cl (market share da Gerdau - líder) ao redor de 60\%, chegando aproximadamente a $90 \%$ quando a Belgo Mineira é incorporada, e atinge $100 \%$ com a inclusão da Siderúrgica Barra Mansa. O mercado de vergalhões, conseqüentemente, por ser o mais concentrado, apontava para um maior DWL (como comprovado pelos números). O HHI, comparativamente aos demais segmentos, é o mais alto: quase igual a 0,5 .

Em seguida vem o mercado de aços longos, em que a Gerdau é também líder, com aproximadamente $50 \%$ do mercado. O C2, quando a Belgo Mineira é incluída, é de cerca de $80 \%$, e o C3, quando a Villares é incorporada, é de $90 \%$. O seu DWL/Fat., apesar de expressivo, é menor do que o encontrado para o mercado de vergalhões, um subgrupo de aços planos. O HHI é de aproximadamente 0,35 . Neste grupo, além de vergalhões, há outros tipos de aço cujas estruturas de mercado são também concentradas. Um exemplo é o dos tubos sem costura, ofertados por uma única empresa: a Vallourec e Mannesmann Tubos. Outros exemplos são as barras de aço inoxidável e as barras de aço para ferramentas e matrizes, ofertados por duas empresas: Gerdau e Villares Metal.

Por fim, tem-se o mercado de aços planos. Apesar de demonstrar a menor concentração entre os três, também apresenta poucos rivais. Tal como o mercado de aços longos há, neste, seis empresas. O Cl é de aproximadamente 35\%, liderado pela CST em quantidade e pela CSN em faturamento. O C2, quando a COSIPA é incorporada, passa para $70 \%$, em média, e o C3, para 90\%. O HHI é, em média, de 0,25. O mercado, não obstante se mostrar o menos concentrado, comparativamente ao de aços longos, também apresenta submercados com pouca rivalidade, tais como os das chapas inoxidáveis e as siliciosas, das quais a Acesita é a única ofertante. Em chapas cromadas e folhas de flandres, a monopolista é a CSN.

\section{CONCLUSÃO}

O mercado siderúrgico é concentrado, em nível internacional, e não seria diferente no Brasil. Este fato é comprovado pelo baixo número de empresas em cada segmento do setor, o que torna possível às firmas exercerem poder de mercado. Os perdedores são

19 As participações aqui descritas são aproximadas e basearam-se nos valores de receita líquida operacional obtidos no site da CVM. 
os consumidores, e os ganhadores, as firmas. A evidência maior dessa elevada probabilidade foi a condenação, pelo CADE, de um caso de infração à ordem econômica cometido por empresas no mercado do aço planos (vale dizer, menos concentrado do que o de aços longos) no ano de 1999, mas que ainda vem sendo analisado pelo Poder Judiciário. Essa realidade (que pode ser acelerada com pesquisas sobre o setor) e também a ausência de estudos quantitativos nesta área foram os maiores motivadores para a realização deste trabalho.

Este ensaio teve, assim, dois objetivos fundamentais. O primeiro foi calcular o valor da perda do bem-estar do setor siderúrgico no Brasil, uma vez que este mensura a perda, para a sociedade, do poder de mercado das empresas em uma determinada indústria. A fundamentação teórica teve como base o trabalho de Daskin (1991), que apresenta uma fórmula de cálculo para mercados oligopolizados, motivado pelo modelo pioneiro de Harberger (1954).

Foi necessário, para alcançar a meta, estimar a equação de demanda por aço e a sua elasticidade-preço, pois não se encontrou pesquisa com esta estimação. Este, portanto, foi o segundo objetivo do presente estudo, que também pode ser utilizado como insumo para a análise ou para a elaboração de políticas públicas relacionadas ao setor em tela. Como metodologia, optou-se, primeiro, por averiguar a existência de raiz unitária, de acordo com o teste $\mathrm{ADF}$. Como a hipótese desta raiz foi rejeitada, o modelo não foi estimado pela técnica de co-integração. A equação de demanda foi, assim, estimada pelo método da variável instrumental.

Os dois objetos de estudo deste ensaio são, portanto, os primeiros a serem realizados, do ponto de vista quantitativo, para o Brasil, o que faz com que o mesmo sirva de referência para outros estudos que venham a ser feitos nessa área, sobre este mercado, em particular. Uma possível extensão, por exemplo, seria a realização do cálculo do DWL para os diversos setores da economia brasileira, a fim de se obter a perda de bem-estar em relação ao PIB do País. Outra possibilidade seria a estimação da demanda e o cálculo do DWL para subgrupos de cada tipo de aço (o mercado de vergalhões, por exemplo).

Os resultados sugerem que a elasticidade-preço da demanda é de -0,14. Além disso, com relação à perda de bem-estar social, se forem considerados os dados relativos aos anos de 2000 e 2001, pode-se concluir que o DWL não é desprezível, como Harberger (1954) defendia em suas estimaçôes.

As conclusões desta pesquisa mostram que há, em 2000 e 2001, uma perda, para a sociedade, de aproximadamente R $\$ 3,5$ bilhões (a preços médios destes anos). Comparativamente, o mercado de vergalhóes apresenta o maior DWL, 31\% com referência ao faturamento do seu setor, seguido pelo de aços longos (26\%), que, por sua vez, é se- 
guido pelo mercado de aços planos (22\%). A interpretação do valor porcentual de

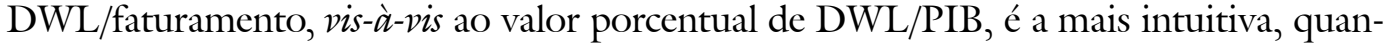
do se quer observar a perda relativa ao tamanho de cada mercado. $\mathrm{O}$ outro valor, no entanto, mostra a perda, para a sociedade, relativa ao tamanho da economia. As duas informações, em conjunto, são, assim, de igual importância.

\section{REFERÊNCIAS BIBLIOGRÁFICAS}

Abbott, A. J.; Lawler, K. A.; Armistead, C. The UK demand for steel. Applied Economics, v. 31, n. 11, p. 1299-1302, 1999.

Bain, J. Relation of profit rate to industry concentration: American manufacturing, 1936-40. Quarterly Journal of Economics, v. 65, n. 3, p. 293-324, 1951.

Bershanhan. Empirical studies of industries with market power. In: Schmalansee, R.; Willig, R. (eds.), Handbook of industrial organization. Amsterdam: NorthHolland, 1989.

Clarke, R.; Davies, S. Market structure and price-cost margins. Economica, v. 49, p. 227-287, 1982.

Cowling, K.; Mueller, D. The social costs of monopoly. Economic Journal, p. 727$748,1978$.

. The social costs of monopoly power revisited. Economic Journal, p. 721$725,1981$.

Cowling, K.; Waterson, M. Price-cost margins and market structure. Economica, v. 43, p. 267-274, 1976.

Cysne, R. P.; Issler, J. V.; Resende, M.; Wyllie, R. Demanda por cervejas no Brasil: um estudo econométrico. Pesquisa e Planejamento Econômico, v. 31, p. 249-68, 2001.

Daskin, A. J. Perda do bem-estar in oligopoly: a new approach. Southern Economic Journal, v. 58, n. 1, p. 171-185, 1991.

Dickson, V. A. Conjectural variation elasticities and concentration. Economic Letters, p. 281-85, 1981.

Dixit, A.; Stern, N. Oligopoly and welfare. European Economic Review, v. 19, p. 123$143,1982$.

Enders, W. Applied econometric time series. $1^{\text {a }}$ ed. Nova York: John Wiley \& Sons, Inc., 1995.

Fergunson, P. R.; Fergunson, G. J. Industrial economics: issues and perspectives. London: MacMillan, 1994.

Geroski, P. A.; Philips, L.; Ulph, A. Oligopoly, competition and welfare: some recent developments. Journal of Industrial Economics, v. 33, p. 369-86, 1985.

Greene, W. H. Econometric analysis. $3^{\mathrm{a}}$ ed. Estados Unidos: Prentice Hall Inc., 1997. 
Harberger, A. C. Monopoly and resource allocation. American Economic Review, p. 77-87, Maio 1954.

Holt, C. A. On the use of profit data to estimate the social costs of monopoly power in an oligopoly. 1982, p. 283-289.

Instituto Brasileiro de Siderurgia. Anuário Estatístico. 2002.

Johansen, S. Statistical analysis of cointegrating vectors. Journal of Economic Dynamics and Control, v. 12, p. 231-254, 1988.

. Estimation and hypothesis testing of cointegrating vectors in Gaussian vector autoregressive models. Econometrica, v. 59, p. 1551-80, 1991.

Johansen, S., Juselius, K. Maximum likelihood estimation and inference on cointegration with application to the demand for money. Oxford Bulletin of Economics and Statistics, v. 52, p. 169-209, 1990.

Mas-Colell, A.; Whinston, M.; Green, J. Microeconomic theory. Oxford University Press, 1995.

Masson, R. T.; Shaanan, J. Social costs of oligopoly and the value of competition. Economic Journal, p. 520-535, 1984.

Mendes de Paula, G. Novos métodos de previsão de demanda de aço. 2002. Mimeografado.

Scherer, F; Ross, D. Industrial market structure and economic performance. Boston: Houghton Mifflin, 1990.

Schmalansee, R. Inter-industry studies of structure and performance. In: Schmalansee, R.; Willig, R. (eds.), Handbook of industrial organization. Amsterdam: North-Holland, 1989.

Varian, H. R. Microeconomic analysis. Third edition. W.W. Norton \& Company Inc., 1992.

Vasconcellos, M. A. S.; Alves, D. (coords.), Manual de econometria: nível intermediário. $1^{a}$ edição. Equipe de Professores da USP, São Paulo: Atlas, 2000.

Wyllie, R. L. Mercado de cerveja no Brasil: um estudo econométrico. 2001. Dissertação (Mestrado), EPGE/FGV, Rio de Janeiro. 
APENNDICE

1. Equivalência entre as Equações (2) e (3)

Fórmula apresentada em Daskin (1991):

$$
D W L=\frac{1}{2} \Pi^{2} \eta \cdot\left(R^{-1}\right)
$$

Fórmula apresentada em Fergunson e Fergunson (1994):

$$
D W L=\frac{1}{2}\left(\frac{\Delta p}{p}\right)^{2} \eta \cdot R
$$

Considerando:

$$
\begin{aligned}
& \Delta p=p_{m}-p_{c} \\
& p_{c}=C m g=\text { Custo Marginal } \\
& \Delta p=p_{m}-C m g
\end{aligned}
$$

$\log$ :

$$
\Pi=\Delta p \cdot Q
$$

Multiplicando e dividindo o lado direito de (2) por Q, obtém-se:

$$
\begin{aligned}
D W L & =\frac{1}{2}(\Delta p)^{2} \cdot Q^{2} \eta \cdot \frac{1}{P \cdot Q} \\
D W L & =\frac{1}{2} \Pi^{2} \eta \cdot\left(R^{-1}\right)
\end{aligned}
$$

\section{Derivação da Condição de Primeira Ordem}

Supõe-se que, para a firma i, a sua elasticidade entre as quantidades vendidas de qualquer firma k com relação a ela, firma i, é constante. Isto é, supõe-se que 
$\eta_{i}=\frac{\Delta \% q_{k}}{\Delta \% q_{i}}=\frac{d q_{k}}{d q_{i}} * \frac{q_{i}}{q_{k}}=\alpha_{i}, \forall \mathrm{k} .{ }^{20}$ Conseqüentemente, a condição de primeira ordem (CPO), para cada firma i, é:

$$
\begin{aligned}
& \frac{d \Pi}{d q_{i}}=P(Q)+q_{i} \frac{d P(Q)}{d Q} \frac{d Q}{d q_{i}}-C m g_{i}=0 \\
& \frac{d \Pi}{d q_{i}}=P(Q)+q_{i} \frac{d P(Q)}{d Q}\left[\frac{d q_{i}}{d q_{i}}+\frac{d q_{1}}{d q_{i}}+\ldots+\frac{d q_{N-1}}{d q_{i}}\right]-C m g_{i}=0 \\
& \frac{d \Pi}{d q_{i}}=P(Q)+q_{i} \frac{d P(Q)}{d Q}\left[1+\sum_{i \neq k=1}^{N-1} \frac{d q_{k}}{d q_{i}}\right]-C m g_{i}=0 \\
& P(Q)+\frac{P(Q) * Q}{P(Q) *} * q_{i} \frac{d P(Q)}{d Q} *\left[1+\sum_{i \neq k=1}^{N-1} \frac{d q_{k}}{d q_{i}}\right]-C m g_{i}=0 \\
& P(Q)+P(Q) * \frac{q_{i}}{Q} *\left[\frac{Q}{P(Q) *} \frac{d P(Q)}{d Q}\right] *\left[1+\sum_{i \neq k=1}^{N-1} \alpha_{i} \frac{q_{k}}{q_{i}}\right]-C m g_{i}=0 \\
& P(Q)\left\{1+S_{i} *\left[-\frac{1}{|\varepsilon|}\right] *\left[1+\sum_{i \neq k=1}^{N-1} \alpha_{i} * \frac{q_{k}}{Q} * \frac{Q}{q_{i}}\right]\right\}-C m g_{i}=0 \\
& P(Q)\left\{1-\frac{S_{i}}{|\varepsilon|} *\left[1+\sum_{i \neq k=1}^{N-1} \alpha_{i} * \frac{q_{k}}{Q} * \frac{1}{S_{i}}\right]\right\}-C m g_{i}=0 \\
& P(Q)\left\{1-\frac{S_{i}}{|\varepsilon|} *\left[1+\frac{\alpha_{i}}{S_{i}} \sum_{i \neq k=1}^{N-1} \frac{q_{k}}{Q}\right]\right\}-C m g_{i}=0 \\
& P(Q)\left\{1-\frac{S_{i}}{|\varepsilon|} *\left[1+\frac{\alpha_{i}}{S_{i}}\left(1-S_{i}\right)\right]\right\}-C m g_{i}=0
\end{aligned}
$$

Note que $S_{i}=\frac{q_{i}}{Q}$ é a participação de mercado da firma i, $|\varepsilon|$ é a elasticidade-preço da demanda, e $\sum_{i \neq k=1}^{N-1} \frac{q_{k}}{Q}$ representa a participação de mercado de todas as demais firmas do

20 O modelo exposto foi fundamentado, pela primeira vez, por Cowling e Waterson (1976). Os demais trabalhos teóricos, como o de Clarke e Davies (1982), seguem o mesmo raciocínio destes autores, alterando apenas a forma de modelar a variação conjectural. 
mercado (menos a firma i), ou seja, é igual a $\left(1-S_{i}\right)$. Reescrevendo a equação acima, chega-se, então, à seguinte expressão:

$$
P(Q)\left\{1-\frac{\left[S_{i}+\alpha_{i}\left(1-S_{i}\right)\right]}{\varepsilon}\right\}-C_{i}=0
$$

3. Derivação do Excedente do Produtor (EP) e do Excedente do Consumidor (EC)

$$
\begin{aligned}
E P & =\sum_{k=1}^{N} \Pi_{k}=\sum_{k=1}^{N}\left[P(Q)-C_{i}\right]^{*} q_{i}=\sum_{k=1}^{N} \frac{\left[P(Q)-C_{i}\right]}{P(Q)} * \frac{q_{i}}{Q} *[P(Q) * Q] \\
E C & =\int_{P_{C P}}^{P_{O}} A u^{-\varepsilon} d u, \varepsilon \neq 1 \\
E C & =\left.\frac{A u^{(1-\varepsilon)}}{(1-\varepsilon)}\right|_{P_{C P}} ^{P_{O}}= \\
E C & =\frac{A P(Q)_{O}^{(1-\varepsilon)}}{(1-\varepsilon)}-\frac{A P(Q)_{C P}{ }^{(1-\varepsilon)}}{(1-\varepsilon)}= \\
E C= & \frac{A P(Q)_{O}{ }^{-\varepsilon} P(Q)_{O}}{(1-\varepsilon)}\left[1-\left(\frac{P(Q)_{C P}}{P(Q)_{O}}\right)^{(1-\varepsilon)}\right]= \\
E C= & \frac{Q_{O} P(Q)_{O}}{(1-\varepsilon)}\left[1-\left(\frac{P(Q)_{C P}}{P(Q)_{O}}\right)^{(1-\varepsilon)}\right]= \\
E C= & \frac{R T(Q)_{O}}{(1-\varepsilon)}\left[1-\left(\frac{P(Q)_{C P}}{P(Q)_{O}}\right)^{(1-\varepsilon)}\right]
\end{aligned}
$$

Onde o subscrito $\mathrm{CP}$ refere-se à concorrência perfeita, e o subscrito $\mathrm{O}$, a oligopólio. Usando o índice de Lerner e supondo que $C^{*}=\operatorname{Min}_{i}^{*}=P(Q)_{C P}$, tem-se que $L_{i}=\frac{P(Q)_{O}-P(Q)_{C P}}{P(Q)_{O}}=1-\frac{P(Q)_{C P}}{P(Q)_{O}}$, ou melhor, $\frac{P(Q)_{C P}}{P(Q)_{O}}=1-\mathrm{L}_{\mathrm{i}}$. 
4. Nomes das Empresas

\begin{tabular}{ll}
\hline \multicolumn{2}{c}{ Empresas por tipo de aço } \\
\hline Aços Planos & 1 Acesita \\
& 2 Gerdau + Açominas \\
& 3 CSN \\
& 4 COSIPA \\
& 5 CST \\
& 6 Usiminas \\
& 1 Acesita \\
& 2 Gerdau + Açominas \\
Aços Longos & 3 Belgo \\
& 4 Barra Mansa \\
& 5 Villares e VM \\
& 6 Vallourec e Mannesmann \\
& 1 Gerdau + Açominas \\
& 2 Belgo \\
Vergalhões & 3 Barra Mansa \\
\end{tabular}

\section{Resultados EC, EP e DWL (em $R \$$ de cada periodo)}

EC, EP E DWL EM RS\$ DE CADA PERÍODO / ELASTICIDADE = - 0,14

\begin{tabular}{|c|c|c|c|c|}
\hline \multirow[b]{2}{*}{ Aços Planos } & \multicolumn{2}{|c|}{ Sem importação } & \multicolumn{2}{|c|}{ Com importação } \\
\hline & 2000 & 2001 & 2000 & 2001 \\
\hline $\mathrm{EC}$ & $2.106 .716 .325,12$ & $1.334 .535 .057,20$ & $2.265 .286 .371,10$ & $1.434 .983 .932,47$ \\
\hline EP & $269.119 .824,66$ & $(442.401 .707,56)$ & $237.180 .123,37$ & $(411.433 .588,03)$ \\
\hline DWL & 1.837.596.500,46 & $1.776 .936 .764,76$ & 2.028.106.247,73 & $1.846 .417 .520,50$ \\
\hline Fat. & $8.166 .169 .831,60$ & $8.739 .826 .194,78$ & $8.780 .827 .775,91$ & $9.397 .662 .575,03$ \\
\hline PIB & $1.101 .255 .078 .000,00$ & 1.198.736.188.000,00 & $1.101 .255 .078 .000,00$ & $1.198 .736 .188 .000,00$ \\
\hline DWL/Fat. & $22,50 \%$ & $20,33 \%$ & $23,10 \%$ & $19,65 \%$ \\
\hline \multirow[t]{2}{*}{ DWL/PIB } & $0,167 \%$ & $0,148 \%$ & $0,184 \%$ & $0,154 \%$ \\
\hline & \multicolumn{2}{|c|}{ Sem importação } & \multicolumn{2}{|c|}{ Com importação } \\
\hline Aços Longos & 2000 & 2001 & 2000 & 2001 \\
\hline $\mathrm{EC}$ & $2.044 .615 .034,87$ & $2.735 .446 .901,99$ & $2.152 .226 .352,49$ & $2.879 .417 .791,57$ \\
\hline EP & $564.845 .409,99$ & $1.104 .775 .298,15$ & $530.894 .037,90$ & $1.025 .118 .365,19$ \\
\hline DWL & $1.479 .769 .624,88$ & $1.630 .671 .603,83$ & 1.621.332.314,59 & $1.854 .299 .426,38$ \\
\hline Fat. & $5.866 .583 .466,40$ & $6.191 .156 .635,02$ & $6.175 .351 .017,27$ & $6.517 .006 .984,23$ \\
\hline $\mathrm{PIB}$ & 1.101.255.078.000,00 & 1.198.736.188.000,00 & $1.101 .255 .078 .000,00$ & 1.198.736.188.000,00 \\
\hline DWL/Fat. & $25,22 \%$ & $26,34 \%$ & $26,25 \%$ & $28,45 \%$ \\
\hline \multirow[t]{2}{*}{ DWL/PIB } & $0,134 \%$ & $0,136 \%$ & $0,147 \%$ & $0,155 \%$ \\
\hline & \multicolumn{2}{|c|}{ Sem importação } & \multicolumn{2}{|c|}{ Com importação } \\
\hline Vergalhão & 2000 & 2001 & 2000 & 2001 \\
\hline $\mathrm{EC}$ & $760.251 .625,84$ & $873.578 .416,26$ & $800.264 .869,30$ & $919.556 .227,64$ \\
\hline EP & $246.495 .908,26$ & $310.735 .189,10$ & $234.171 .112,85$ & $295.198 .429,65$ \\
\hline DWL & $513.755 .717,57$ & $562.843 .227,15$ & $566.093 .756,45$ & $624.357 .797,99$ \\
\hline Fat. & $1.552 .597 .116,05$ & $2.037 .108 .515,47$ & $1.634 .312 .753,73$ & $2.144 .324 .753,12$ \\
\hline PIB & 1.101.255.078.000,00 & $1.198 .736 .188 .000,00$ & 1.101.255.078.000,00 & 1.198.736.188.000,00 \\
\hline DWL/Fat. & $33,09 \%$ & $27,63 \%$ & $34,64 \%$ & $29,12 \%$ \\
\hline DWL/PIB & $0,047 \%$ & $0,047 \%$ & $0,051 \%$ & $0,052 \%$ \\
\hline
\end{tabular}

